\title{
Isolated Dissection of the Supramesenteric Artery and Conservative Treatment
}

\author{
Takatsugu Yamamoto ${ }^{\mathrm{a}, \mathrm{c}}$, Koichiro Abe ${ }^{\mathrm{a}}$, Hajime Anjiki ${ }^{\mathrm{a}}$, Taro Ishii ${ }^{\mathrm{a}}$, \\ Yasushi Kuyama ${ }^{a}$, Hiroshi Kohtake ${ }^{b}$, Shigeru Furui ${ }^{b}$
}

\begin{abstract}
A 59-year-old male with hypertension admitted to our hospital because of abdominal pain. Visceral angiography revealed stenosis of the supramesenteric artery due to isolated dissection. The symptom disappeared soon with conservative treatment, then he was discharged and prophylactic low-dose aspirin was initiated. Seven months later he underwent angiography again due to postprandial abdominal discomfort, which showed improvement of the stenosis. Conservative treatment with aspirin can be a choice in case of visceral artery dissection when ischemic symptoms do not exist.
\end{abstract}

Keywords: Supramesenteric artery; Dissection; Conservative treatment

\section{Introduction}

Spontaneous dissection of the supramesenteric artery (SMA) without associated aortic dissection is a rare condition. Recently, reports of such cases increase in association with advance of diagnostic technique, but the consensus of the treatment has not been established yet. Here we report on a case of SMA dissection which was treated conservatively.

Manuscript accepted for publication January 27, 2012

${ }^{a}$ Department of Internal Medicine, Teikyo University School of Medicine, 2-11-1 Kaga, Itabashi-ku, 173-8605, Tokyo, Japan

${ }^{\mathrm{b}}$ Department of Radiology, Teikyo University School of

Medicine, 2-11-1 Kaga, Itabashi-ku, 173-8605, Tokyo, Japan

${ }^{\mathrm{c}}$ Corresponding author: Takatsugu Yamamoto.

Email: ymmtmze@zpost.plala.or.jp

doi: $10.4021 / \mathrm{jmc} 536 \mathrm{w}$

\section{Case Report}

A 59-year-old male noticed abdominal pain suddenly after hinge drinking in March, 2005. He had taken antihypertensive agents for 10 years. When he visited to our hospital (Teikyo University Hospital, Tokyo, Japan), the vital signs, the abdominal $\mathrm{x}$-ray and electrocardiogram did not show any abnormal finding. The laboratory data demonstrated slight abnormality in coagulation test. Since the symptom continued, he underwent computed tomography indicating isolated dissection of SMA. Visceral angiography demonstrated dissection and stenosis with apparent pressure discrepancy between proximal and distal portion of SMA, without ischemic change (Fig. 1). Surgical or radiological intervention was considered, but the patient chose conservative treatment with oral prescription of low-dose aspirin $100 \mathrm{mg} /$ day and antihypertensive medicines. The abdominal symptom disappeared soon and did not appear again even after initiating oral intake, so he was discharged. The patient began to feel postprandial abdominal discomfort 7 months later. Visceral angiography was performed again, which showed significant

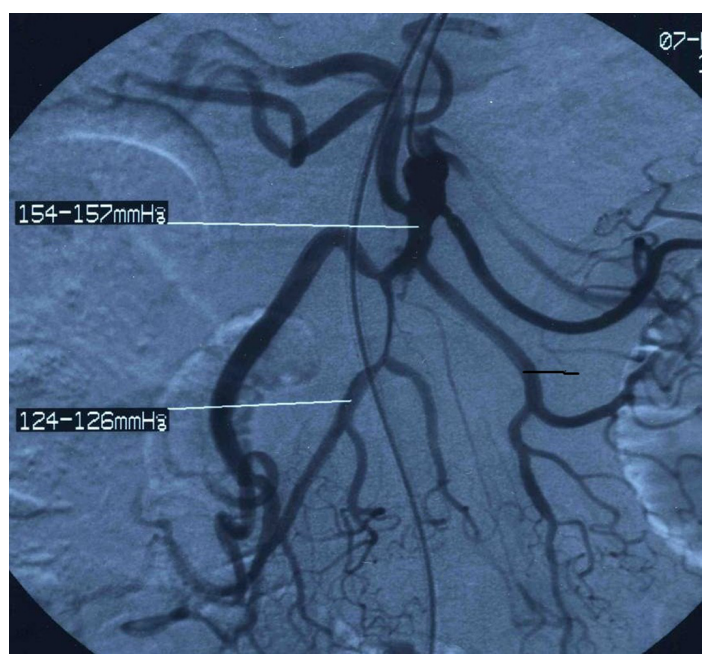

Figure 1. First visceral angiography. Stenosis of the supramesenteric artery with obvious pressure discrepancy was seen. 


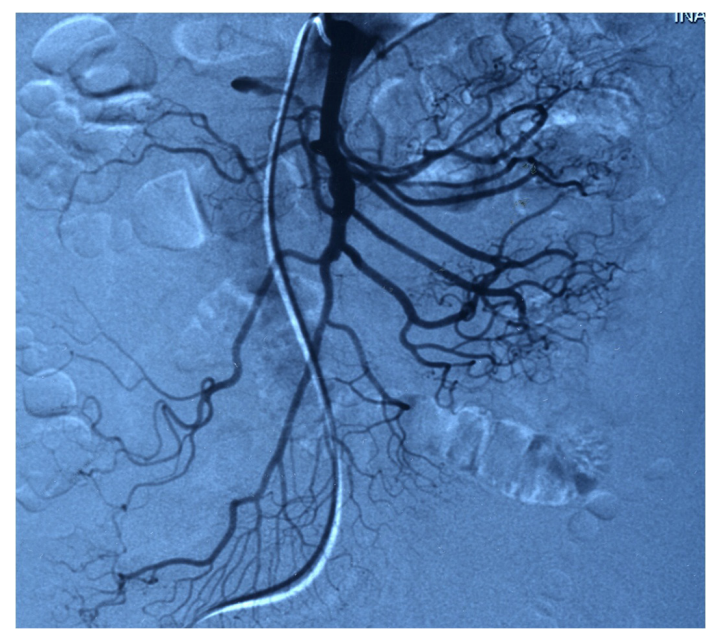

Figure 2. Second angiography. Stenosis has been improved.

improvement of the lesion (Fig. 2). He has been well with prescription of aspirin and antihypertensive drugs.

\section{Discussion}

Spontaneous dissection of the visceral artery is life-threatening condition which can cause intestinal ischemia or rupture of the artery. Emergent surgical or radiological intervention may be considered in case which those critical complications are suspected. However, recent reports indicate that emergent intervention is not mandatory when patients are asymptomatic or with only mild symptom without ischemic change $[1,2]$. Medical management of the condition includes antihypertensive and antiplatelet therapy that prevents expansion of dissection and formation of thrombus. In the present case, the conservative treatment was successful.

Here we reported on a case of spontaneous isolated dissection of SMA which was successfully treated conservatively with antihypertensive medicine and aspirin. Because patients having visceral arterial dissection will increase in association with progressing aged society in developed countries including Japan, clinicians should know the disease and the possibility of conservative treatment with antihypertensive and antiplatelet agents.

\section{References}

1. Zhang WW, Killeen JD, Chiriano J, Bianchi C, Teruya $\mathrm{TH}$, Abou-Zamzam AM. Management of symptomatic spontaneous isolated visceral artery dissection: is emergent intervention mandatory? Ann Vasc Surg. 2009;23(1):90-94.

2. Amabile P, Ouaissi M, Cohen S, Piquet P. Conservative treatment of spontaneous and isolated dissection of mesenteric arteries. Ann Vasc Surg. 2009;23(6):738-744. 